\title{
Dextran Penetration Through Nonkeratinized and Keratinized Epithelia in Monkeys*
}

\author{
Carlos E. Nasjleti† and Raul G. Caffesseł
}

Accepted for publication 22 August 1983

\begin{abstract}
THE PURPOSE OF THIS STUDY was to determine if polysaccharide dextrans would pass through intact-nonkeratinized and induced-keratinized sulcular epithelia in monkeys. Dextran penetration through normally keratinized oral gingival epithelium also was evaluated in the same gingival specimens. Each of three Rhesus monkeys received a thorough prophylaxis 1 week prior to the experiment. During this week, the monkeys also received daily IV injections of Achromycin. After the antibiotic treatment, the teeth were polished and cleaned with a rubber cup using prophylactic paste for 2 consecutive months, as follows: (1) the right maxillary and mandibular teeth received daily prophylaxes on weekdays and (2) the left maxillary and mandibular teeth received one prophylaxis weekly. These frequencies of plaque removal on one-half of the mouth maintained clinically healthy gingiva and produced keratinization of the sulcular epithelium. At the end of the 2-month prophylaxes, a 5\% solution of dextrans derived from Leuconostoc mesenteroides was applied topically to the gingiva once daily for 3 consecutive weeks. During this time, the monkeys continued having dental prophylaxes following the previous time schedule. The study showed that inducedkeratinized sulcular epithelium as well as normally keratinized oral gingival epithelium resisted penetration of dextrans, whereas intact-nonkeratinized sulcular epithelium apparently lacked a surface layer resistant to penetration.
\end{abstract}

The literature on penetration of macromolecules as well as smaller molecules through both keratinized and nonkeratinized sulcular epithelia is controversial. The controversy relates to differences in the molecular weight of the substances tested, in the species used and in the systems used to test for penetration. Many studies have documented penetration through the sulcular or oral epithelia in animals ${ }^{1-11}$ and humans. ${ }^{12} \mathrm{~A}$ few, have not supported such a contention. ${ }^{13-15}$

The same discrepancy is evident when studies dealing with dextran penetration through gingival epithelia are evaluated. Neuman et al. ${ }^{16}$ reported that tritium-labeled dextran, with a molecular weight of 200,000 to 300,000 , penetrated the healthy gingival sulci of beagle dogs and induced chronic gingival inflammation. Tolo and Jonsen ${ }^{17}$ also demonstrated penetration of tritiated dextran, molecular weight 70,000 , through nonkeratinized rabbit oral mucosa. Similarly, Vogel et al. ${ }^{18}$ showed penetration of carbon-14-labeled dextran through non-

\footnotetext{
* This investigation was supported by the Medical Research Division of the Veterans Administration.

+ Coordinator, Dental Research Program, Veterans Administration Medical Center, Ann Arbor, Ml 48105.

$\ddagger$ Professor and Chairman. Department of Periodontics. The University of Michigan School of Dentistry, Ann Arbor, MI 48109.
}

keratinized and induced-keratinized sulcular epithelia in human gingival specimens. Contrary to these studies, Gaffar et al. ${ }^{19}$ did not observe penetration of streptococcal dextrans per se through the epithelial surfaces of rat gingiva. Prior treatment of these surfaces with streptococcal hyaluronidase, however, allowed penetration of the polysaccharide, resulting in destructive changes in the rat gingiva.

Polysaccharide dextrans are inert molecules which pass selectively through tissue membranes by active energy-consuming transport processes, and they are slowly broken down by mammalian tissues. ${ }^{20}$ Bacterial polysaccharides are known to be present in significant quantities in dental plaque. Ten per cent of the dry weight of dental plaque is composed of the extracellular polysaccharide dextran. ${ }^{21}$ It has also been mentioned that dental plaque, in part, consists of a component antigenically similar to the dextran produced by Leuconostoc mesenteroides. ${ }^{22}$ Dextran from Leuconostoc mesenteroides can induce local chronic inflammation when injected subcutaneously in experimental animals. ${ }^{23}$ Dextrans have been recommended as tracer molecules for permeability studies. ${ }^{24}$

The purpose of the present study was to determine if polysaccharide dextrans would pass through intact-nonkeratinized and induced-keratinized sulcular epithelia 
in monkeys. Normally keratinized oral gingival epithelium was also evaluated for dextran penetration in the same gingival specimens.

\section{MATERIALS AND METHODS}

Three healthy young adult male Rhesus monkeys (Macaca mulatta) with full and intact dentitions were used. They were sedated by an intramuscular injection of ketamine hydrochloride $20 \mathrm{mg} / \mathrm{kg}$ body weight and anesthetized with sodium pentobarbital intravenously, $50 \mathrm{mg} / \mathrm{ml}$. Following an initial clinical examination, all teeth were thoroughly scaled and polished 1 week before the experiment began, in order to remove plaque and calculus and minimize gingival inflammation. During this pre-experimental week, each monkey also received a daily IV injection of Achromycin ${ }^{(x)}$ (tetracycline hydrochloride) at the maximum safe dosage of $20 \mathrm{mg} /$ $\mathrm{kg}$ body weight. For 2 consecutive months after this preliminary week, the teeth were polished and cleaned with a rubber cup using $\mathrm{NuPro}^{(k)}$ prophylactic paste, according to the following schedule: (1) the right maxillary and mandibular teeth received a prophylaxis on each weekday and (2) the left maxillary and mandibular teeth received one prophylaxis weekly (Friday). These frequencies of plaque removal were selected based on previous studies. ${ }^{25-29}$

At the end of this 2-month period, a $5 \%$ solution of dextrans derived from Leuconostoc mesenteroides was applied topically to the gingiva once daily for 3 consecutive weeks (except Saturday and Sunday), as follows: (1) fluorescein isothiocyanate-dextran ${ }^{*}$ in physiologic saline solution, molecular weight (mol wt) 67,000, was applied to the buccal gingiva of the maxillary canines, premolars and molars, bilaterally; and (2) clinical grade dextran $\dagger$ in physiologic saline solution, mol wt 70,000, was applied to the buccal gingiva of mandibular canines, premolars and molars, also bilaterally. These topical applications were made dropwise, with disposable Pasteur pipettes, over the marginal gingiva of each tooth. Five applications, one every 3 minutes, were made on each gingival test site. In addition, small aliquots of the dextran solutions were injected into the attached gingiva of maxillary and mandibular incisor teeth. These injections were performed in order to obtain gingival specimens to serve as tissue controls for the determination of the dextran solutions and were done 1 hour before gingival biopsies were taken at the end of the experiment. During the 3 weeks of dextran applications, the monkeys continued having dental prophylaxes following the same schedule as the previous 2 months.

Under anesthesia, gingival biopsies were taken from the buccal areas of each of the experimental sites. Biopsy specimens were removed by means of two ver-

* Sigma Chemical Company, St. Louis, MO.

$\dagger$ Fisher Scientific Products, Pittsburgh, PA. tical releasing incisions and a horizontal incision connecting the apical ends of the vertical incisions. A third vertical incision divided the biopsy. The gingiva was elevated toward the coronal portion and the attachment was dissected from the tooth surface. One-half of each biopsy was fixed in $10 \%$ buffered formalin, embedded in paraffin, sectioned at 6- $\mu$ intervals and stained with one of the following: Ehrlich's acid hematoxylin and eosin, Mallory's connective tissue stain as modified by Ayoub and Shklar ${ }^{30}$ and Rhodamine B. ${ }^{31}$ The other half of the biopsy specimen was fixed in $100 \%$ ethanol to preserve water-soluble dextran, and subsequently processed for paraffin embedment, sectioned at $6 \mu$, and stained with alcoholic periodic acid Schiff (PAS) and aqueous PAS to detect dextran in tissue according to the technique of Mowry and Millican. ${ }^{32}$

For histologic evaluation, four zones were considered: Zones A, B and C representing the crestal, middle and cervical thirds of the sulcular epithelium, respectively; and Zone D representing the oral gingival epithelium (Fig. 1). Histologic evaluation included: (1) presence or absence of a keratinized layer on the epithelial surfaces and (2) presence or absence of dextran in the gingival tissues. For this, one microscopic field in each zone was evaluated. This field, representing at $100 \times$ magnification a rectangle of $100 \times 68 \mu \mathrm{m}$, was positioned so that the basal cell layer divided the field into two segments, with the connective tissue occupying approximately two thirds of the field and the epithelium occupying the remaining one third. Penetration through the junctional epithelium was also evaluated.

Forty microscopic sections were evaluated for each of the experimental and control sites, 240 per monkey, for a total of 720 sections. These sections were evaluated under a Carl Zeiss binocular photo-microscope. $\neq$ Tissues treated with fluorescent dextran as well as Rhodamine B-stained specimens were examined using the same microscope and the fluorescent light attachments: a HBO 200 mercury lamp, a BG 12 primary filter and a $530 \mathrm{~nm}$ interference filter.

\section{RESULTS}

Table 1 summarizes the results. When dextran, labeled or unlabeled, was applied topically to intact nonkeratinized sulcular epithelium, it passed through the epithelium into the underlying connective tissue. Dextran spread deeply into connective tissue, both laterally to the sulcular epithelium and apically to the junctional epithelium. Dextran penetration was observed mainly in sulcular epithelium Zone C; however, occasionally it was also depicted in Zone B. When dextran permeated through nonkeratinized epithelium, it induced widening of the epithelial intercellular spaces and vacuolation of the underlying connective tissue. Figure 2 illustrates normal gingival tissue of the monkey, show-

$\ddagger$ Okekochen. West Germany. 


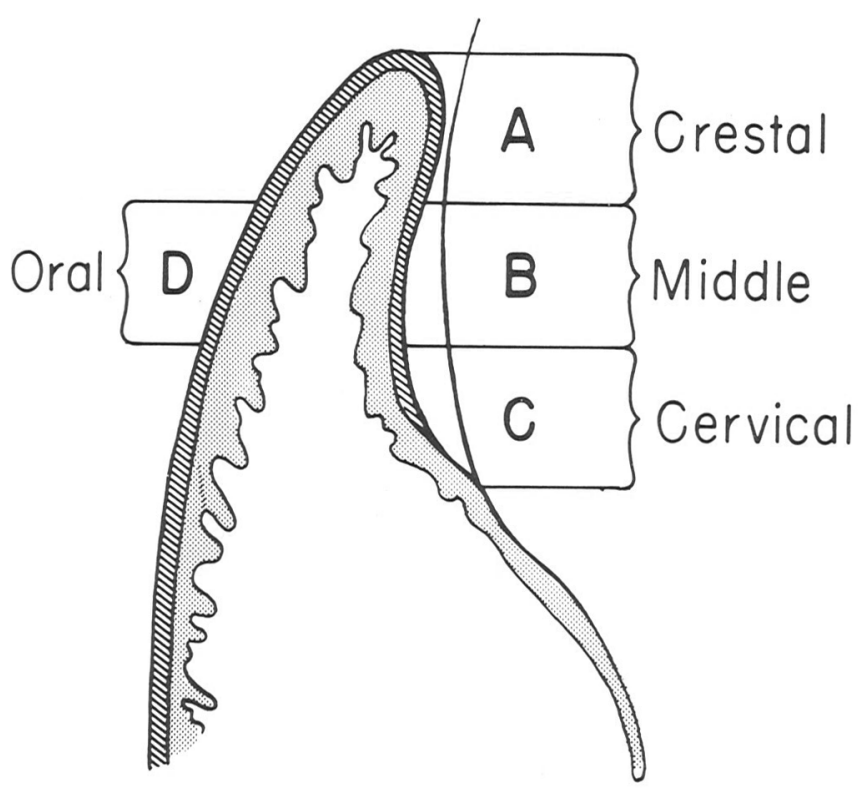

Figure 1. Diagram showing the location of the four zones, $A, B, C$ and $D$. where dextran penetration was evaluated.

\section{Table 1}

Degrees of Dextran Penetration in Gingival Epithelia

\begin{tabular}{lcc} 
Zone & Keratinized* & Non-keratinized* \\
\hline A & 0 & 0 \\
B & 0 & + \\
C & 0 & ++ \\
JE $\dagger$ & + & +++ \\
D & 0 & 0
\end{tabular}

* $0=$ no penetration, $+=$ minimal penetration, $++=$ slight penetration. $+++=$ moderate penetration.

$\dagger \mathrm{JE}=$ Junctional epithelium. ing both nonkeratinized sulcular and keratinized oral gingival epithelia, and Figure 3 demonstrates fluorescent-dextran within epithelial and connective tissues.

When dextran was applied to induced-keratinized sulcular epithelium as well as to normally keratinized oral gingival epithelium (Fig. 4), it failed to penetrate and remained on the outer surface of the keratinized epithelia (Figs. 5 and 6). It showed minimal penetration,

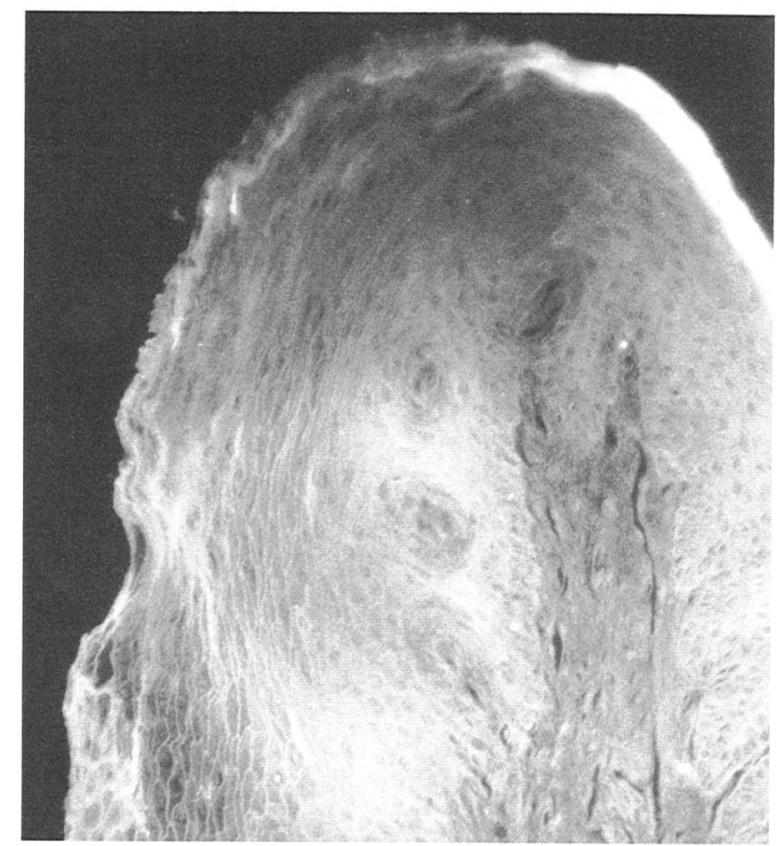

Figure 2. Normal gingival tissue of Rhesus monkey. Keratinized oral gingival epithelium. as demonstrated by fluorescent stain. stopped at the gingival margin (Rhodamine $B$ stain, original magnification $\times$ 100).

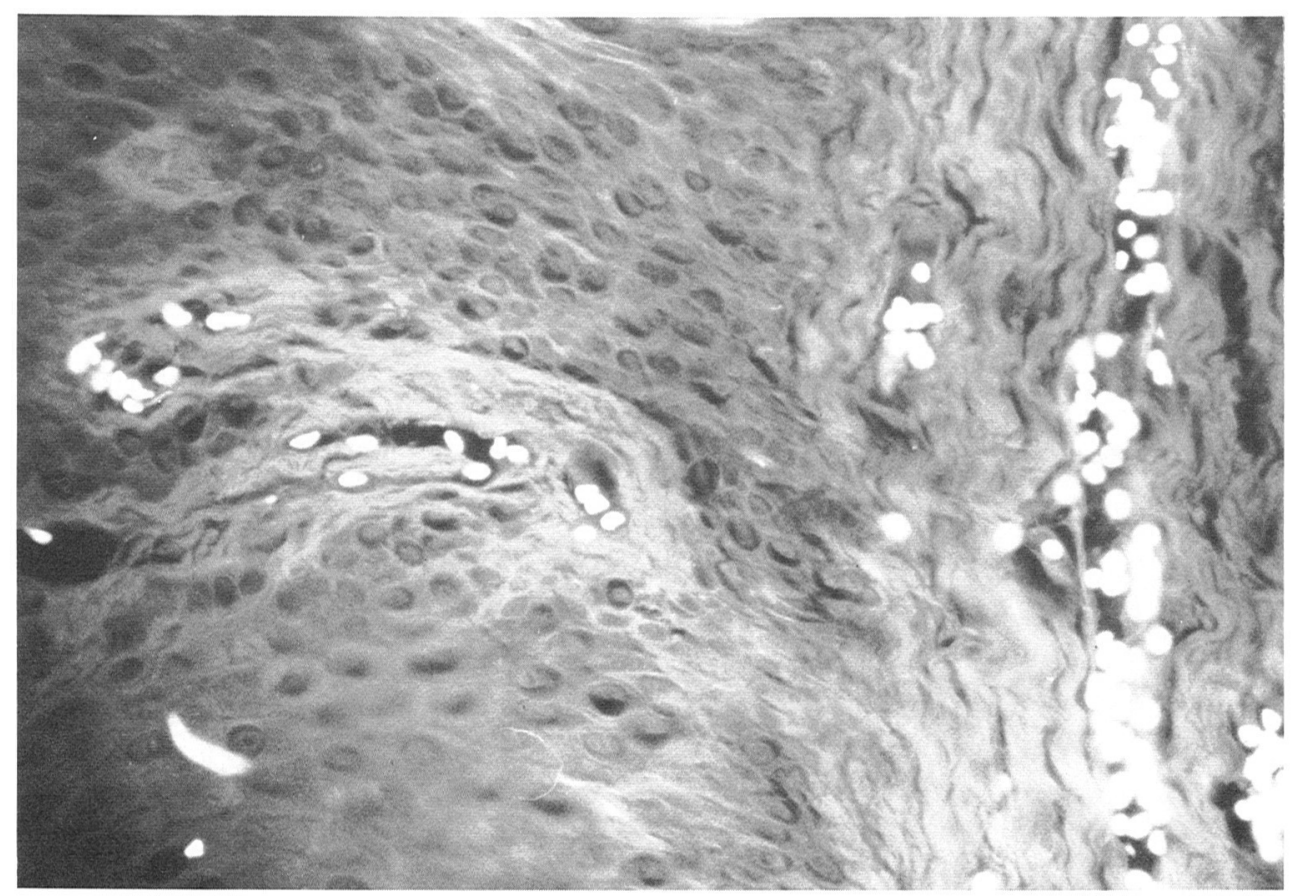

Figure 3. Topically applied fluorescent-dextran penetrated through nonkeratinized sulcular epithelium into the underlying connective tissue (Mallory's stain, original magnification $\times 250$ ). 


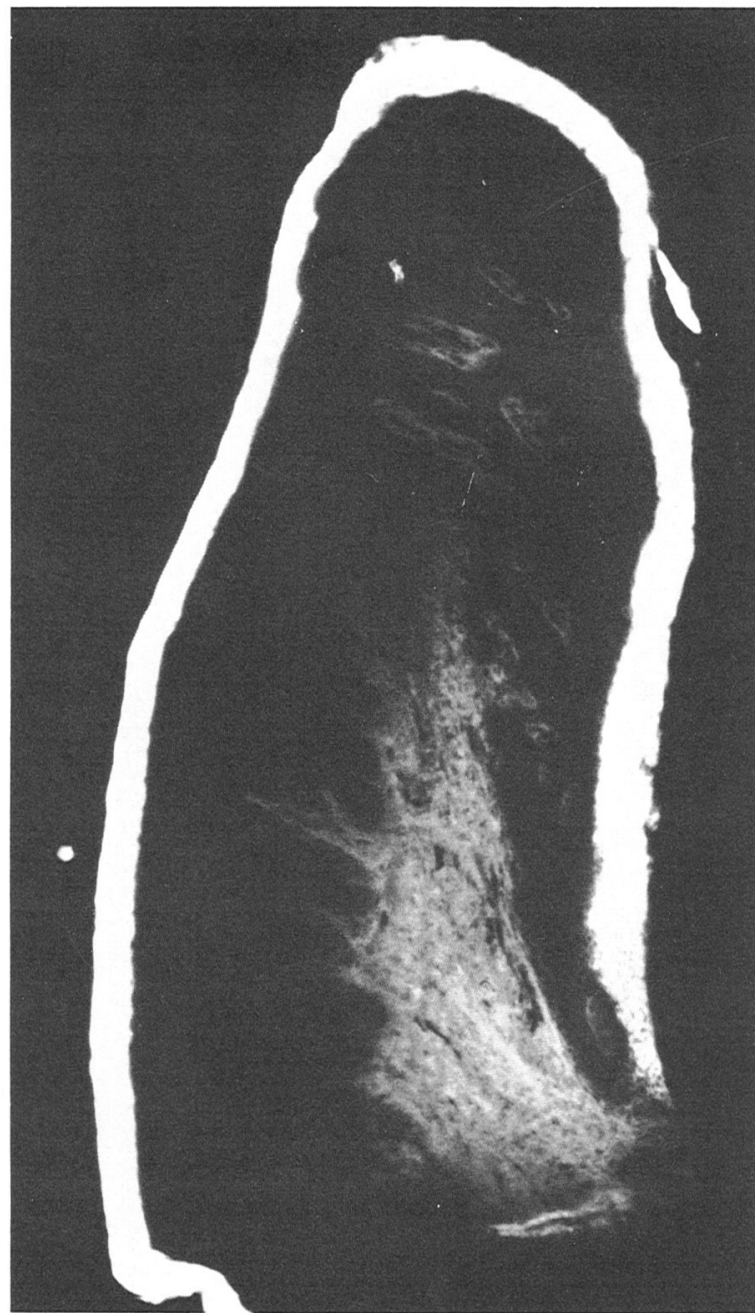

Figure 4. Induced-keratinized sulcular epithelium as demonstrated by. fluorescent microscopy: Thick keratin band covered sulcular epithelium (Rhodamine B stain original magnification $\times 100)$.

however, at the bottom of the sulcus, through the junctional epithelium. Also, in areas of lacerated or injured keratinized epithelium. it was possible to see dextran penetration (Fig. 7). Gingival tissues injected with solutions of dextran clearly showed its presence within epithelial (Fig. 8) and connective tissues (Fig. 9).

\section{DISCUSSION}

The polysaccharide dextran, as used in the present study, had the ability to penetrate intact-nonkeratinized sulcular epithelium, widening the epithelial intercellular spaces and vacuolizing the underlying connective tissues. However, dextran penetrated neither the induced-keratinized sulcular epithelium nor the normally keratinized oral gingival epithelium. Minimal dextran penetration was observed at the bottom of the sulcus, or when the sulcular epithelium was not completely keratinized, or when keratinized epithelia was injured on its outer surface.

The widening of the epithelial intercellular spaces was similar to that reported previously following topical application of the enzyme hyaluronidase to intact-nonkeratinized sulcular epithelium in monkeys. " According to McDougall, ${ }^{4}$ such a widening of the intercellular spaces seemed to be a general and early response to the presence of noxious substances. Thilander ${ }^{33.34}$ reported that the intercellular spaces of the human sulcular epithelium widened after topical application of a subject's own leukocyte homogenate. Freeman et al. ${ }^{35}$ described relatively large intercellular spaces as a characteristic feature of chronically inflamed human sulcular epithelia.

How dextran passed through intact-nonkeratinized sulcular epithelium cannot be ascertained by the present study. However, of the known possible mechanisms. endocytosis or pinocytosis, active transport and diffusion, ${ }^{36}$ the most likely is diffusion. The observations of McDougall ${ }^{4}$ suggested that transport of noxious substances across sulcular epithelium might occur by diffusion through the epithelial intercellular spaces. According to Tolo and Jonsen, ${ }^{17}$ macromolecular penetration through sulcular epithelium in vivo was probably influenced by the presence of mucosal secretions, im-

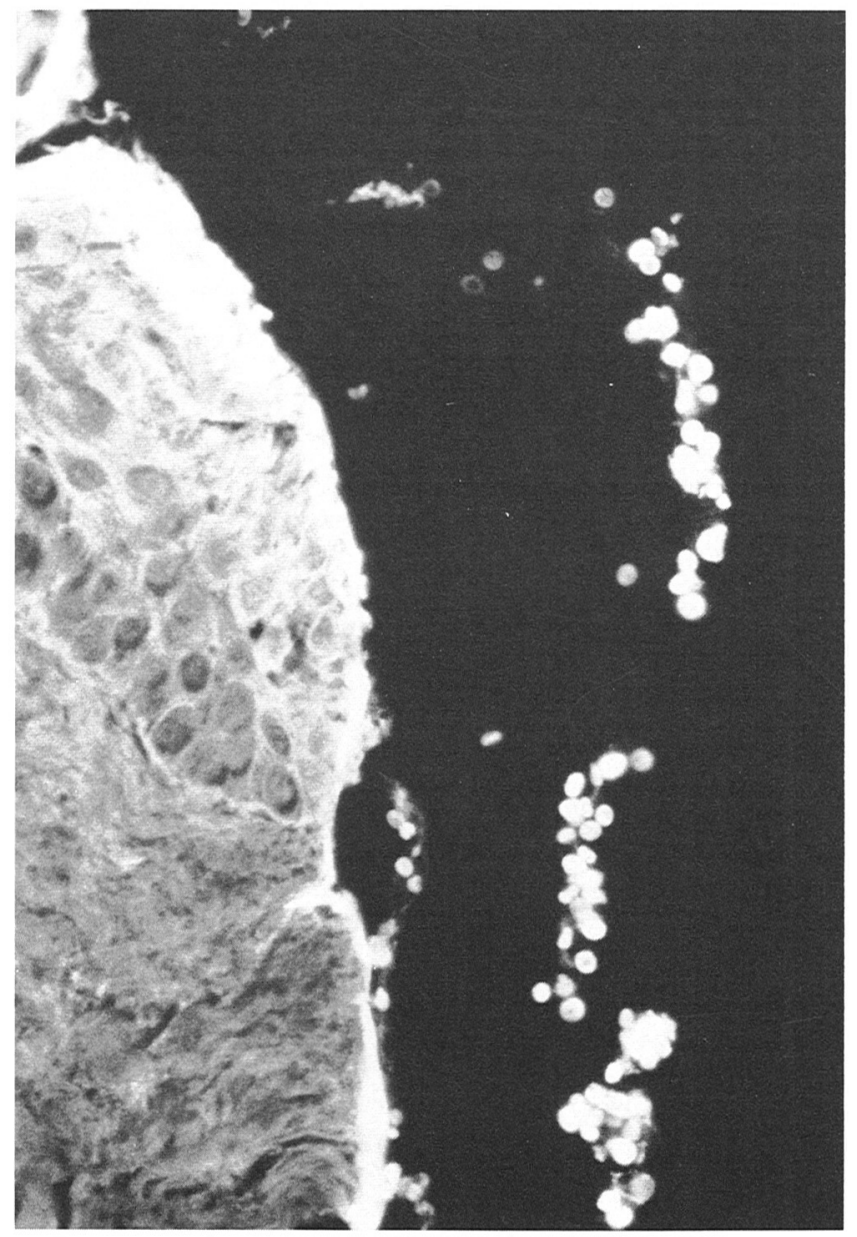

Figure 5. Fluorescent-dextran did not permeate through inducedkeratinized sulcular epithelium. Dextran band can be seen on keratinized sulcular epithelial surface (Rhodamine B stain, original magnification $\times 250$ ) 


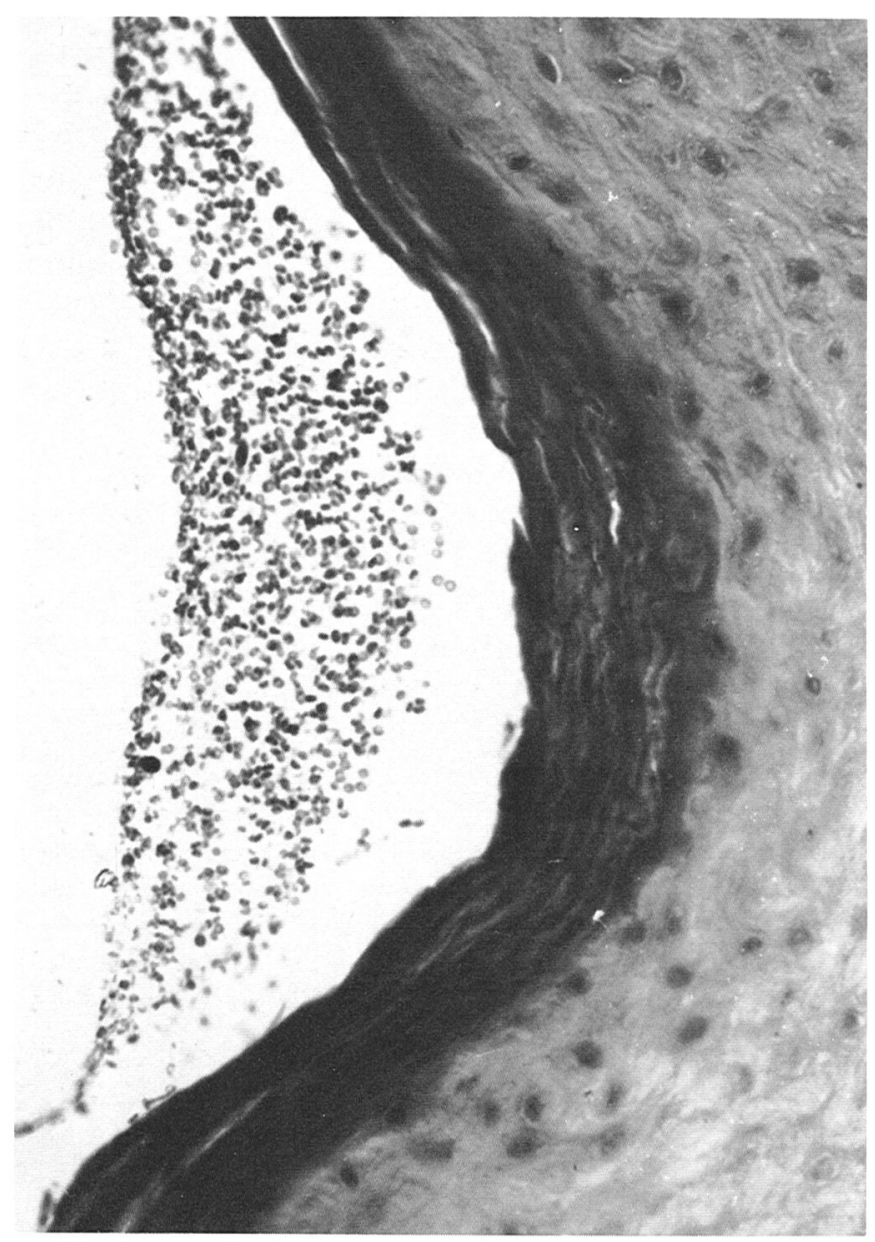

Figure 6. Clinical grade dextran did not penetrate normally keratinized oral gingival epithelium (PAS, Schiff's stain, original magnification $\times 250$ ).

mune reactions and the activity of a relatively large number of neutrophils, macrophages and lymphocytes.

The finding regarding dextran penetration through nonkeratinized sulcular epithelium is consistent with earlier work by Neuman et al. ${ }^{16}$ and Tolo and Jonsen. ${ }^{17}$ Neuman et al. ${ }^{16}$ placed dextrans on clinically healthy gingival tissues of beagle dogs once a day for 21 days. Dextrans were able to penetrate the sulcular epithelium, enter the underlying connective tissue and cause chronic inflammation. Tolo and Jonsen ${ }^{17}$ studied the in vitro penetration of dextrans across rabbit oral mucosa. They demonstrated that nonkeratinized epithelium, in the course of 120 minutes, permitted penetration of dextrans with a molecular weight of approximately 70,000. They also showed that penetration of radioactive dextrans was markedly decreased by inhibition of cell glycolysis and oxidative phosphorylation, as well as by incubation at $4^{\circ} \mathrm{C}$.

In the present study the lack of polysaccharide penetration seems to have been due mainly to the increasing sulcular epithelial keratinization. These results tend to substantiate previous reports from this laboratory. ${ }^{25.37}$ Caffesse et al. ${ }^{25}$ reported penetration of triti-

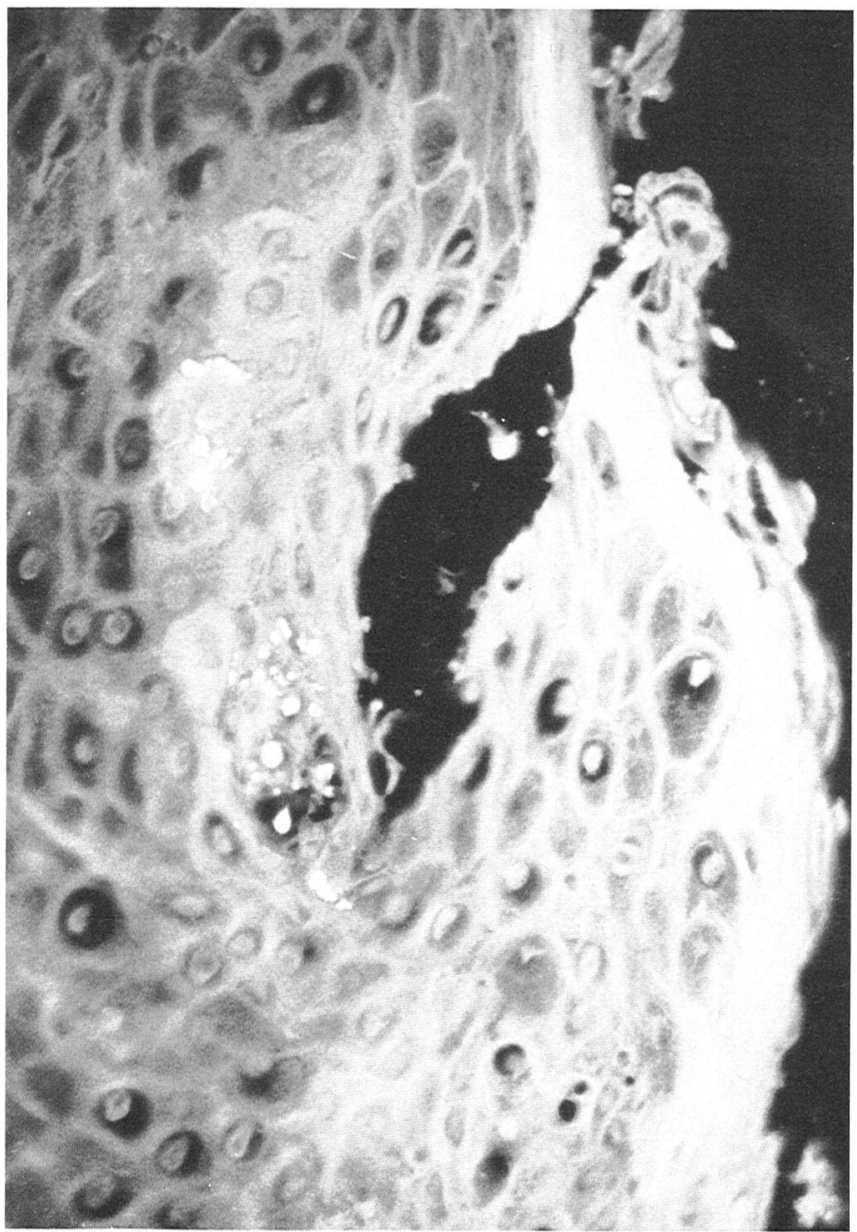

Figure 7. Minimal dextran penetration was observed in areas adjac'ent to injured or lacerated keratinized epithelia (Rhodamine B stain. original magnification $\times 250$ ).

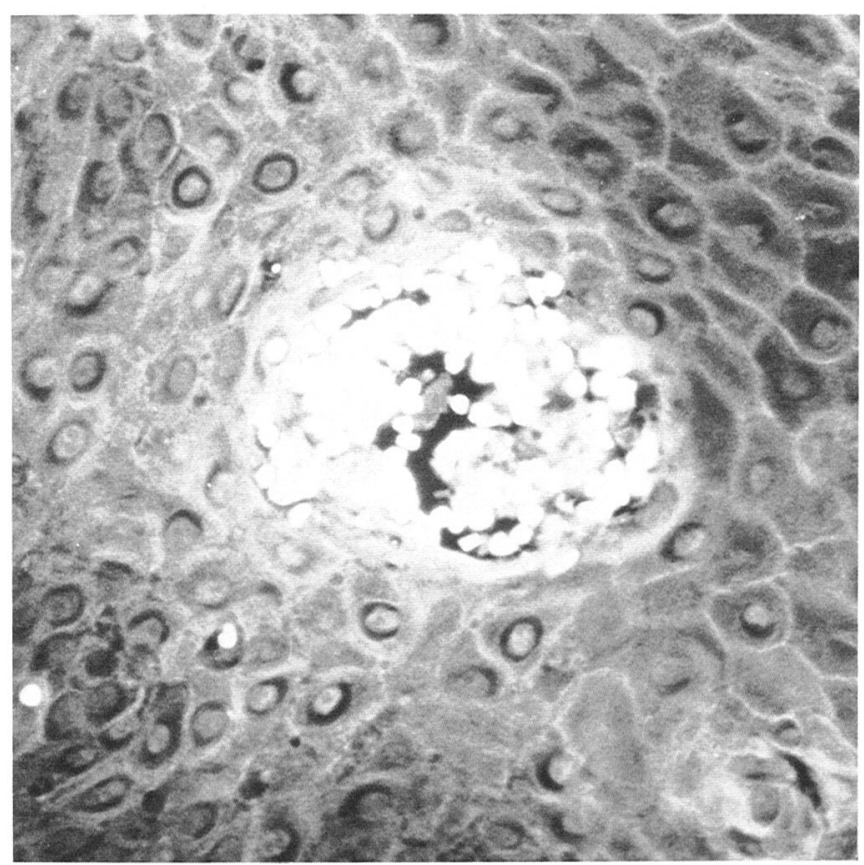

Figure 8. Control specimen. Injected fluorescent-dextran (Mallory's stain, original magnification $\times 250$ ). 


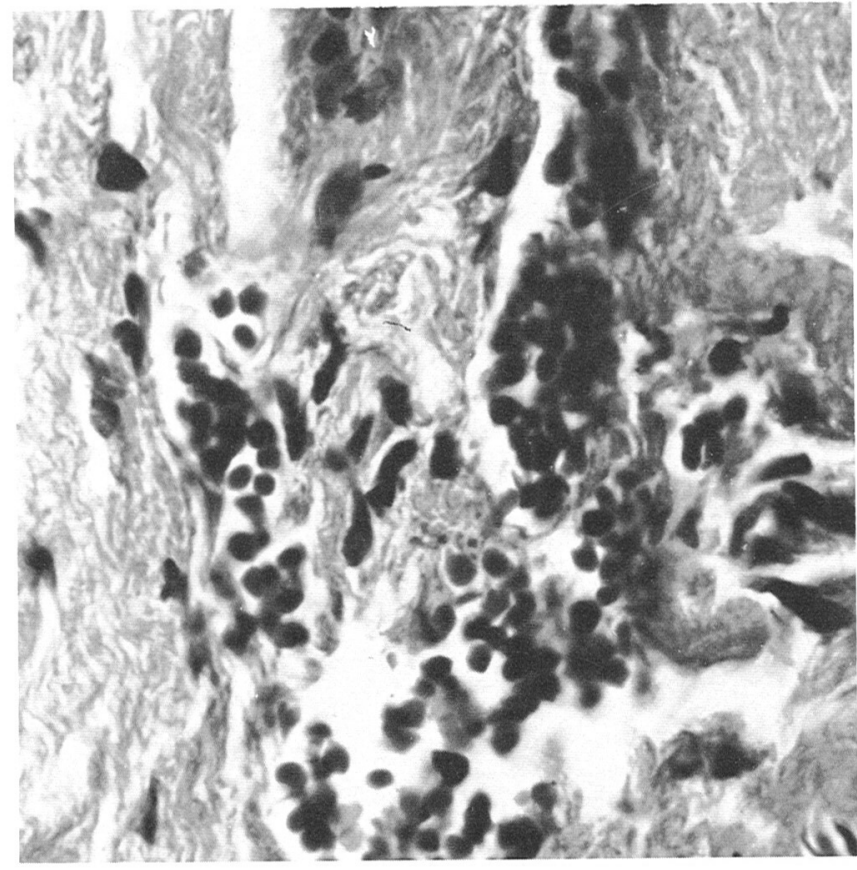

Figure 9. Control specimen. Injected clinical grade dextran (PAS, Schiffss stain, original magnification $\times 250)$.

ated thymidine through intact-nonkeratinized sulcular epithelium in monkeys. However, no penetration was observed through induced-keratinized epithelium. Kristoffersen et al. ${ }^{37}$ studied by transmission electron microscopy intact-nonkeratinized sulcular epithelium, induced-keratinized sulcular epithelium and normally keratinized oral gingival epithelium. The results showed that the ultrastructural characteristics of both intact sulcular epithelium and oral gingival epithelium were similar to those reported by other investigators. ${ }^{38-40}$ Ultrastructural observations of induced-keratinized sulcular epithelium confirmed earlier histologic studies which have shown that following rubber cup prophylaxes, the epithelium becomes parakeratinized or orthokeratinized. ${ }^{28.29}$ A number of other structural changes were also observed, some of which suggested that the prophylaxes given might have resulted in the formation of a more efficient permeability barrier in the sulcular area.

Recently, Vogel et al. ${ }^{18}$ studied the effect of intrasulcular brushing on sulcular permeability in humans. One group cleaned their teeth daily, using an intrasulcular technique, whereas the other group used an extrasulcular technique. After 49 days, gingival biopsies were taken and evaluated in vitro for permeability using a microperfusion technique. The intrasulcular group demonstrated a significantly higher degree of sulcular epithelial keratinization. It also showed that dextran, used for evaluating permeability, was able to permeate through not only nonkeratinized sulcular epithelium but also induced-keratinized sulcular epithelium. No relationship between the degree of sulcular epithelial keratinization and permeability of the tissue was found.
It may be that rubber cup prophylaxes induce more keratin than intrasulcular brushing does, and consequently, that a more effective barrier to dextran penetration could be obtained. ${ }^{28.29}$

In summary, induced-keratinized sulcular epithelium as well as normally keratinized oral gingival epithelium have been found to resist penetration of topically applied dextran in monkeys, while intact-nonkeratinized sulcular epithelium apparently lacks a surface layer resistant to penetration.

\section{REFERENCES}

1. Tolo, K.: A study of permeability of gingival pocket epithelium to albumin in Guinea pigs and Norwegian pigs. Arch Oral Biol 16: $881,1971$.

2. Jensen, R. L., and Folke, L. E. A.: The passage of exogenous tritiated thymidine through the sulcular epithelium (Abstr. 349). Int Assoc Dent Res Program and Abstr, p 136, 1970.

3. Jensen. R. L., and Folke, L. E. A.: The passage of exogenous tritiated thymidine into gingival tissues. $J$ Periodontol 45: 786, 1974.

4. McDougall, W. A.: Penetration pathways of a topically applied foreign protein into rat gingiva. J Periodont Res 6: 89, 1971.

5. Montgomery, E. H., and Ranney, R. R.: Endotoxin: an initiation of acute gingival inflammation (Abstr. 163). Int Assoc Dent Res Program and $1 \mathrm{hstr}, \mathrm{p} 99,1973$.

6. Schwartz. J.. Stinson. F., and Parker. R.: The passage of tritiated bacterial endotoxin across intact gingival crevicular epithelium. $J$ Periodontol 43: 270, 1972

7. Schultz-Haudt, S., Dewar, M., and Bibby, B.: Effects of hyaluronidase on human gingival epithelium. Science 117: 653, 1953.

8. Smith, F., and Ramfjord, S.: Hyaluronidase applied to the gingiva of rhesus monkeys. $J$ Periodontol 44: 361, 1973.

9. Murphy, P. J.. and Stallard. R. E.: An altered gingival attachment epithelium: A result of the enzyme hyaluronidase. Periodontics 6: 105,1967

10. Stallard. R. E., and Awwa, A.: The effect of alterations in external environment on dentogingival junction. J Dent Res 48: 667 . 1969.

11. Caffesse. R. G., and Nasjleti, C. E.: Enzymatic penetration through intact sulcular epithelium. J Periodontol 47: 391. 1976.

12. Fine, D. H., Pechersky, J. L., and McKibben, D. H.: The penetration of human gingival sulcular tissue by carbon particles. Arch Oral Biol 14: 1117, 1969.

13. Gibson, W. A., and Shannon, I. L.: Stimulation with carbon particles of bacterial infiltration of human gingival tissues. Periodontics 3: 57,1965

14. Squier, C. A.: The permeability of keratinized and nonkeratinized oral epithelium to horseradish peroxidase. J Ultrastruct Res 43: 160, 1973.

15. Rizzo, A. A.: Adsorption of bacterial endotoxins into rabbit gingival pocket tissue. Periodontics 6: 65, 1968.

16. Neuman. S. M., Kenney, E. B., and Traurig. H.: The effect of topical application of dextran on the gingiva of beagle dogs. $J$ Periodontol 47: 171, 1976.

17. Tolo, K., and Jonsen, J.: In vitro penetration of tritiated dextrans through rabbit oral mucosa. Arch Oral Biol 20: 419. 1975.

18. Vogel, R. I., Alfano, M. J., and Manhold. J. H.: The effect of intrasulcular brushing on sulcular epithelial permeability. J Periodontol 52: 244, 1981.

19. Gaffar, A., Coleman. E. J., and Marcussen, H. W.: Penetration of dental plaque components into gingiva: sequential topical treatments with hyaluronidase and streptococcal polysaccharide in rats. $J$ Periodontol 52: 197, 1981 .

20. Sisson, W. B., and Oldendorf, W. H.: Brain distribution spaces of mannitol- $-{ }^{3} \mathrm{H}$, inulin $-{ }^{14} \mathrm{C}$, and dextran- $-{ }^{14} \mathrm{C}$ in the rat. Am $J$ Physiol 221: $214,1971$. 
21. Wood, J. M.: The stateof hexose sugar in human dental plaque and its metabolism by the plaque bacteria. Arch Oral Biol 14: 161, 1969.

22. Gibbons, R. J., and Banghardt, S. B.: Synthesis of extracellular dextran by cariogenic bacteria and its presence in human dental plaque. Arch Oral Biol 12: 11, 1967.

23. Spector, W. G.: Factors in chronicity in skin inflammation. $J$ Pathol 96: 205, 1968.

24. Arturson, G., and Granath, K.: Dextrans as test molecules in studies of the functional ultrastructure of biological membranes. Clin Chim Acta 37: 309, 1972

25. Caffesse, R. G., Bye, F. L., Nasjleti, C. E., and Castelli, W. A.: Penetrability of keratinized sulcular epithelium (Abstr. 351). Am Assoc Dent Res Program and Abstr, p 355, 1980.

26. Caffesse, R. G., Karring, T., and Nasjleti, C. E.: Keratinization potential of sulcular epithelium. J Periodontol 48: 140, 1977.

27. Caffesse, R. G., Kornman, K. S., and Nasjleti, C. E.: The effect of intensive antibacterial therapy on the sulcular environment in monkeys. Inflammation, mitotic activity and keratinization of the sulcular epithelium. $J$ Periodontol 51: $155,1980$.

28. Bye, F. L., Caffesse, R. G., and Nasjleti, C. E.: The effect of different plaque control modalities on the keratinizing potential of the sulcular epithelium in monkeys. $J$ Periodontol 51: 632, 1980.

29. Caffesse, R. G., Nasjleti, C. E., Kowalski, C. J., and Castelli, W. A.: The effect of mechanical stimulation on the keratinization of sulcular epithelium. J Periodontol 53: 89, 1982.

30. Ayoub, $P_{A}$ and Shklar, G.: A modification of the Mallory connective tissue stain as a stain for keratin. Oral Surg 16: 580, 1963.

31. Clausen, F., and Dabelsteen, E.: Increase in sensitivity of the
Rhodamine B method for keratinization by the use of fluorescent light. Acta Pathol Microbiol Scand 77: 169, 1969.

32. Mowry, R., and Millican, R.: Histochemical study of the fate of dextran in tissues in the monkey. Am J Pathol 29: 523, 1953.

33. Thilander, H.: The effect of leukocyte enzyme activity on the structure of the pocket epithelium in man. Acta Odontol Scand 21: $432,1963$.

34. Thilander, H.: Epithelial changes in gingivitis. J Periodont Res 3: $303,1968$.

35. Freeman, H. L.. Listgarten, M. A., and Taichman, N. S.: Electron microscopic features of chronically inflamed human gingiva. J Periodont Res 3: 313, 1968.

36. Squier, C. A., and Johnson, N. W.: Permeability of oral mucosa. Br Med Bull 31: 169, 1975.

37. Kristoffersen, E. K., Caffesse, R. G. Nasjleti, C. E., and Kristoffersen, T. $\emptyset$.: Ultrastructural study of induced keratinization in sulcular gingival epithelium in rhesus monkeys. Acta Odontol Scand. 41: 227, 1983.

38. Listgarten, M. A.: Normal development, structure, physiology, and repair of gingival epithelium. Oral Sci Rev 1: 3, 1972.

39. Listgarten, M. A.: The ultrastructure of human gingival epithelium. Am J Anat 114: 49, 1964.

40. Schroeder, H. E., and Thelaide, J.: Electron microscopy of normal human gingival epithelium. J Periodont Res 1: 95, 1966.

Send reprint requests to: Dr. Carlos E. Nasjleti, Dental Research Section. Veterans Administration Medical Center, 2215 Fuller Rd, Ann Arbor, MI 48105 .

\section{Abstracts}

Phenytoin-Induclid Hypierplasia of the Preeruptive Stage. REPORT OF A CASE

Royer, J. E., Hendrickson, D. A., and Scharpf, H. O.

Oral Surg 56: 365, October, 1983

The side effects of the sodium diphenylhydantoinate (Dilantin), which is used in the treatment of seizure disorders, are well known. A case of a severe Dilantin-induced gingival hyperplasia, covering the primary dentition of a 2-year-old boy with 6 to $8 \mathrm{~mm}$ of soft tissue was reported. The patient was hospitalized, and general anesthesia used. Drug therapy was changed from phenobarbital and Dilantin to phenobarbital. Tegretol and Valproate. Electrocautery was used to expose the labial surfaces of the teeth, and the rest of the tissue reduced. Healing was satisfactory within 4 weeks. The modification of the medical treatment and the tissue reduction performed led to a successful result. Naval Regional Dental Center, Box 147. San Diego, (.A 92136

Dr. Serge Dibart

\section{Acchilitated Periodontal Disease in a Patient With Essential. Mixed CRyogiobulinemia}

Woods, S. E. and Goldstein. A. R. Oral Surg 56: 263, September, 1983

A case of a patient with Type III essential mixed cryoglobulinemia and a history of gingival swelling and hemorrhage in conjunction with accelerated periodontal disease was reported. In cases of cryoglobulinemia, there is evidence of serum proteins which have the ability to precipitate reversibly or gel on exposure to cold and redissolve at higher temperatures. The clinical manifestations are mainly purpura, occurring on the lower limbs, thighs, buttocks, abdomen. hands and arms and symptoms which are described as Raynaud's Phenomena. There is a strong likelihood that immune mechanisms are responsible for periodontal destruction in response to bacterial etiology. Upon comparing radiographs for the 32-year-old female patient, there was very little bone loss if any between 1974 and 1979 . A significant change was found upon comparing the radiographs between 1979 and 1981 when a generalized average alveolar bone loss of $4.5 \mathrm{~mm}$ was found. A combination treatment of antibiotics and elimination of local factors by full mouth subgingival debridement, root planing and curettage was performed. Although home care was good, the patient's periodontal condition was clearly unstable. 440 Three Mile Course, Guilford, CT $06437 . \quad$ Dr. Zvi Artzi

\section{Diabetes Incriases Collagienase activity in Extracts of RAT GingIVA AND SKIN}

Ramamurthy, N. S. and Golub, L. M.

$J$ Periodont Res 18: 23, January, 1983

Diabetes mellitus disturbs many metabolic and physiologic processes in host tissue particularly collagenolytic activities. The effect of diabetes on gingival and skin collagen metabolism was demonstrated on rats by inducing diabetes and comparing the results with nondiabetic controls. Either ${ }^{14} \mathrm{C}$-collagen fibrils or Peptide-P was used as a substrate for detecting collagenase digestion products and succinyl(L-alanyl $)_{3}$ - $p$-nitroanilide as a substrate for measuring elastase activities. In the early stage of diabetes, the rats showed no or little collagenase activity similar to the controls, but in the prolonged period of diabetes, they exhibited formation of the collagenase digestion products in extracts of their gingiva and skin. Diabetes increased elastase activity only in extracts of skin. The breakdown of collagen changes the connective tissue of a diabetic leading to impaired wound healing and the development of unusually severe periodontitis. Department of Oral Biology and Pathologl: School of Dental Medicine. Health Sciences C'enter. State University of New York at Stony Brook, Long Island. NY 11794. 